\title{
Testing standard and nonstandard neutrino physics with cosmological data
}

\author{
Elena Giusarma, ${ }^{1}$ Roland de Putter, ${ }^{2,3}$ and Olga Mena ${ }^{1}$ \\ ${ }^{1}$ IFIC, Universidad de Valencia-CSIC, 46071 Valencia, Spain \\ ${ }^{2}$ Jet Propulsion Laboratory, California Institute of Technology, Pasadena, California 91109, USA \\ ${ }^{3}$ California Institute of Technology, Pasadena, California 91125, USA
}

(Received 26 November 2012; published 7 February 2013)

\begin{abstract}
Cosmological constraints on the sum of neutrino masses and on the effective number of neutrino species in standard and nonstandard scenarios are computed using the most recent available cosmological data. Our cosmological data sets include the measurement of the baryonic acoustic oscillation (BAO) feature in the data release 9 CMASS sample of the baryon oscillation spectroscopic survey. We study in detail the different degeneracies among the parameters, as well as the impact of the different data sets used in the analyses. When considering bounds on the sum of the three active neutrino masses, the information in the BAO signal from galaxy clustering measurements is approximately equally powerful as the shape information from the matter power spectrum. The most stringent bound we find is $\sum m_{\nu}<0.32 \mathrm{eV}$ at 95\% C.L. When nonstandard neutrino scenarios with $N_{\text {eff }}$ massless or massive neutrino species are examined, power spectrum shape measurements provide slightly better bounds than the BAO signal only, due to the breaking of parameter degeneracies. Cosmic microwave background data from high multipoles from the South Pole Telescope turns out to be crucial for extracting the number of effective neutrino species. Recent baryon oscillation spectroscopic survey data combined with cosmic microwave background and Hubble Space Telescope measurements give $N_{\text {eff }}=3.66_{-0.21-0.69}^{+0.20+0.73}$ in the massless neutrino scenario, and similar results are obtained in the massive case. The evidence for extra radiation $N_{\text {eff }}>3$ often claimed in the literature therefore remains at the $2 \sigma$ level when considering up-to-date cosmological data sets. Measurements from the Wilkinson Microwave Anisotropy Probe combined with a prior on the Hubble parameter from the Hubble Space Telescope are very powerful in constraining either the sum of the three active neutrino masses or the number of massless neutrino species. If the former two parameters are allowed to freely vary, however, the bounds from the combination of these two cosmological probes get worse by an order of magnitude.
\end{abstract}

DOI: 10.1103/PhysRevD.87.043515

PACS numbers: 98.80.-k, 95.85.Sz, 98.70.Vc, 98.80.Cq

\section{INTRODUCTION}

Over decades, solar, atmospheric, reactor, and accelerator neutrinos have provided compelling evidence for the existence of neutrino oscillations, implying nonzero neutrino masses. Oscillation experiments only provide bounds on the neutrino mass squared differences, and therefore information on the overall scale of the neutrino mass must come from other experiments. Cosmological data provides a tool to test the absolute scale of neutrino masses. Neutrino masses and abundances leave crucial features in several cosmological observables. The amount of primordial relativistic neutrinos affects cosmic microwave background (CMB) anisotropies and nonrelativistic neutrinos in the recent Universe suppress the growth of matter density fluctuations and galaxy clustering. Cosmology can therefore weigh neutrinos, providing an upper bound on the sum of the three active neutrino masses, $\sum m_{\nu} \sim 0.26 \mathrm{eV}$ at 95\% confidence level (C.L.) [1] (see also Refs. [2-4]). The former bound is found when CMB measurements from the Wilkinson Microwave Anisotropy Probe (WMAP) are combined with information on the distribution of galaxies based on a catalog of photometric galaxy redshifts in Sloan Digital Sky Survey III data release 8 (SDSS DR8), and with the most recent measurement of the Hubble parameter from the Hubble Space Telescope (HST). It assumes a flat universe with a cosmological constant, i.e., a $\Lambda \mathrm{CDM}$ cosmology.

In the standard model of elementary particles, there are three active neutrinos. However, there is no fundamental symmetry in nature establishing a definite number of righthanded (sterile) neutrino species. In fact, models with additional $\sim 1 \mathrm{eV}$ massive sterile neutrinos [5] have been introduced to explain short baseline antineutrino data [6] in terms of neutrino oscillations. Up to date cosmological constraints on massive sterile and active neutrino species have been presented in different cosmological scenarios using different data sets, see Refs. [7-12].

In addition, extra relativistic degrees of freedom could also arise from different physics, such as massless sterile neutrino species, axions [13], decay of nonrelativistic matter [14], gravity waves [15], extra dimensions [16], early dark energy [17], or asymmetric dark matter models [18]. Cosmological probes have been extensively exploited in the literature to set bounds on the relativistic energy density of the universe in terms of the effective number of neutrinos $N_{\text {eff }}$ (see Refs. [2-4,19-22]). Measurements of cosmic microwave background (CMB) anisotropies at arc-minute 
TABLE I. Uniform priors for the cosmological parameters considered here.

\begin{tabular}{lc}
\hline \hline Parameter & Prior \\
\hline$\Omega_{b} h^{2}$ & $0.005 \rightarrow 0.1$ \\
$\Omega_{c} h^{2}$ & $0.01 \rightarrow 0.99$ \\
$\Theta_{s}$ & $0.5 \rightarrow 10$ \\
$\tau$ & $0.01 \rightarrow 0.8$ \\
$n_{s}$ & $0.5 \rightarrow 1.5$ \\
$\ln \left(10^{10} A_{s}\right)$ & $2.7 \rightarrow 4$ \\
$f_{\nu}$ & $0 \rightarrow 0.2$ \\
$N_{\text {eff }}$ & $1.047 \rightarrow 10$ \\
\hline \hline
\end{tabular}

angular scales from the South Pole Telescope (SPT) [23] and the Atacama Cosmology Telescope [24], when combined with other cosmological data sets have provided the constraint $N_{\text {eff }}=4.08_{-0.68}^{+0.71}$ at $95 \%$ C.L. [25-28]), showing evidence for $N_{\text {eff }}>0$ at more than $7 \sigma$ and suggesting values higher than those expected in the canonical scenario. If the effective number of neutrino species $N_{\text {eff }}$ is larger than the standard model prediction, i.e., if $N_{\text {eff }}>3.046$ during the big bang nucleosynthesis (BBN) era, the Hubble expansion rate will be larger, causing therefore weak interactions to freeze out earlier. The standard BBN predictions for light element abundances will change. Current analyses of the observed primordial abundances indicate best-fit values of $\Delta N_{\text {eff }} \sim 0.5-0.8[7,9,29,30]$, and bounds $\Delta N_{\text {eff }}<1-2$ at 95\% C.L., depending on the assumptions on the fiducial cosmology, and are consistent with the canonical $\Delta N_{\text {eff }}=0$. Nevertheless, the extra radiation species do not necessarily feature thermal abundances at BBN [31,32] so that the true number of additional species could possibly be larger than the effective number defined in terms of thermal abundances.

Here we follow a general approach and study the constraints on several neutrino scenarios with the most recent available cosmological data. We consider both standard neutrino scenarios with three active massive neutrinos as well as more general, nonstandard schemes with $N_{\text {eff }}$ massless or $N_{\text {eff }}$ massive species. Of the two scenarios with free number of neutrino species, $N_{\text {eff }}$, the latter one is the most realistic and appropriate scenario, since we know observationally that neutrinos have mass. However, this scenario has been explored much less in the literature than the case of $N_{\text {eff }}$ neutrinos with mass fixed to zero (although see, e.g., Refs. [8,10] and the very recent [12]). In fact, we will show that in the scenario with $N_{\text {eff }}$ massive neutrino species, the neutrino constraints from some combinations of cosmological data sets are much worse than in the other two simpler schemes where one of the two parameters is kept fixed. This highlights the danger of neglecting neutrino mass.

In each of the three scenarios analyzed here, we shall study the impact of each data set separately, devoting special attention to parameter degeneracies and to the impact of galaxy clustering data. We shall also study the preferred values of $N_{\text {eff }}$ arising from our different numerical studies. As stated above, $N_{\text {eff }}$ has been claimed to be larger than the standard model expectation in a plethora of cosmological data analyses. We shall see that using upto-date cosmological data, the evidence for $N_{\text {eff }}>3$ still remains at the $2 \sigma$ level. The data sets exploited include recent measurements of the $\mathrm{BAO}$ feature in the data release 9 (DR9; [33]) CMASS sample of the baryon oscillation spectroscopic survey (BOSS) [34,35], which is part of SDSS-III [36] with a median redshift of $z=0.57$ [37].

The structure of the paper is as follows. Section II describes the three standard and nonstandard physics scenarios considered here, summarizing the main effects of the neutrino parameters on the cosmological observables. In Sec. III we describe the data sets used in the numerical analyses and in Sec. IV the constraints on the neutrino thermal abundance $N_{\text {eff }}$ and on the sum of their masses are presented for different combinations of data in the three schemes studied here. We draw our conclusions in Sec. V.

\section{THE MODEL: NEUTRINOS IN COSMOLOGY}

We shall start with the standard scenario, which is made of three massive neutrinos and put constraints on the sum of their masses $\sum m_{\nu}$. We therefore fix $N_{\text {eff }}=3.04$. Current neutrino oscillation data cannot distinguish between the two possible types of ordering of the neutrino mass eigenstates, i.e., information regarding the neutrino mass hierarchy is inaccessible. While the effect of neutrino mass on the CMB is related to the total amount of energy density in the form of massive neutrinos and the individual values of the neutrino masses are irrelevant, the matter power spectrum in principle provides information on the sum of the neutrino masses as well as on the individual values of the $m_{\nu_{i}}$ 's. The nonrelativistic neutrino overdensities cluster at a given redshift $z$ only at scales where the wave number of perturbations is below the neutrino free streaming scale,

$$
k_{f_{s}}(z)=\frac{0.677}{(1+z)^{1 / 2}}\left(\frac{m_{\nu}}{1 \mathrm{eV}}\right) \sqrt{\Omega_{m}} h \mathrm{Mpc}^{-1},
$$

TABLE II. Constraints on $m_{\nu}$ from WMAP data alone and WMAP combined with HST measurements of the Hubble constant, SPT data and SNLS3 data. We have fixed the number of massive neutrinos to 3.046.

\begin{tabular}{lccccc}
\hline \hline & & WMAP & WMAP + HST & WMAP + SPT & WMAP + SNLS3 \\
\hline$\sum m_{\nu}(\mathrm{eV})$ & $68 \%$ C.L. & $<0.60$ & $<0.16$ & $<0.52$ & $<0.24$ \\
& 95\% C.L. & $<1.05$ & $<0.37$ & $<1.07$ & $<0.51$ \\
\hline \hline
\end{tabular}




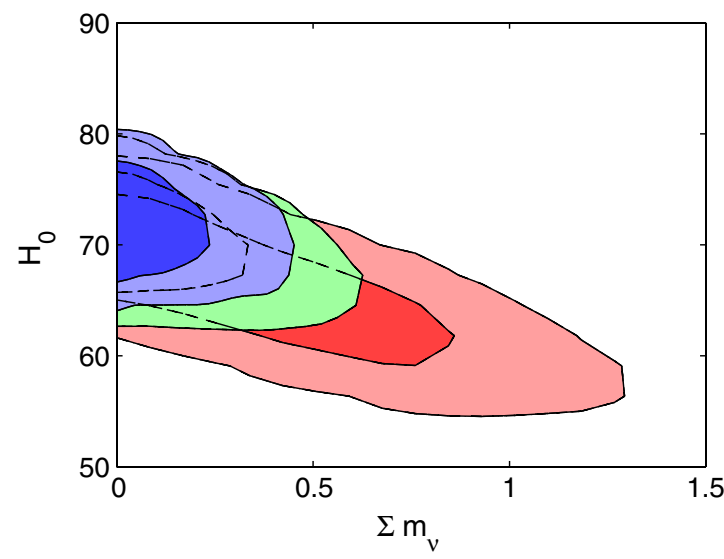

FIG. 1 (color online). The largest red contours show the $68 \%$ and $95 \%$ C.L. constraints in the $\left(\sum m_{\nu}, H_{0}\right)$ plane from our basic WMAP data set. The smallest blue contours show the results from the combination of WMAP and HST data, while the green contours depict the results from the combination of WMAP and SNLS3 data sets. Notice that the strong degeneracy present in the case of WMAP data alone gets alleviated when a prior on $\mathrm{H}_{0}$ from HST data is added in the analysis. SNIa luminosity distance data are unable to independently determine the Hubble constant $H_{0}$ but measure the $\Omega_{m}$ quantity, and, since WMAP measures $\Omega_{m} h^{2}$, the combination of WMAP plus SNIa data is able to determine $H_{0}$.

due to the pressure gradient, which prevents gravitational clustering $\left(\Omega_{m}\right.$ is the ratio of the total matter energy density over the critical density at redshift zero). On spatial scales larger than the free streaming scale $k<k_{f_{s}}$, neutrinos cluster and behave as pressureless matter (dust). Perturbations with comoving wave number larger than the free streaming scale cannot grow due to the large neutrino velocity dispersion. As a consequence, the growth rate of density perturbations decreases and the matter power spectrum is suppressed at $k>k_{f_{s}}$. Therefore, there is a clear signal on the matter power spectrum induced by the presence of neutrino masses. Neutrinos of different masses will have different transition redshifts from relativistic to nonrelativistic behavior, and, in principle, by exploiting the information contained on the matter power spectrum it could be possible to identify the neutrino mass hierarchy and isolate the individual neutrino masses. However, in practice, such a task has been shown to be extremely challenging [38]. In the following, we will assume a degenerate mass spectrum. In the standard scenario with three active massive neutrinos, the parameters considered in the analysis are

$$
\left\{\omega_{b}, \omega_{c}, \Theta_{s}, \tau, n_{s}, \log \left[10^{10} A_{s}\right], \sum m_{\nu}\right\},
$$

where $\omega_{b} \equiv \Omega_{b} h^{2}$ and $\omega_{c} \equiv \Omega_{c} h^{2}$ are the physical baryon and cold dark matter densities, $\Theta_{s}$ is the ratio between the sound horizon and the angular diameter distance at decoupling, $\tau$ is the optical depth, $n_{s}$ is the scalar spectral index, $A_{s}$ is the amplitude of the primordial spectrum, and $\sum m_{\nu}$ is the sum of the active neutrino masses.

Next, we explore nonstandard neutrino scenarios in which the effective number of thermalized species is parametrized by $N_{\text {eff }}$. As commonly assumed in the literature, we start here assuming a massless neutrino scenario with $N_{\text {eff }}$ massless species. One of the main effects of $N_{\text {eff }}$ on the CMB temperature anisotropies arises from the change of the epoch of the radiation matter equality, shifting therefore the location of the CMB acoustic peaks. This position is given by the so-called acoustic scale $\theta_{A}$, which reads

$$
\theta_{A}=\frac{r_{s}\left(z_{\mathrm{rec}}\right)}{r_{\theta}\left(z_{\mathrm{rec}}\right)}
$$

where $r_{\theta}\left(z_{\text {rec }}\right)$ and $r_{s}\left(z_{\text {rec }}\right)$ are the comoving angular diameter distance to the last scattering surface and the sound horizon at the recombination epoch $z_{\text {rec }}$, respectively. Although $r_{\theta}\left(z_{\text {rec }}\right)$ almost remains the same for different values of $N_{\text {eff }}, r_{s}\left(z_{\text {rec }}\right)$ becomes smaller when $N_{\text {eff }}$ is increased. Therefore, the positions of acoustic peaks are shifted to higher multipoles (smaller angular scales) if the value of $N_{\text {eff }}$ is increased. However, this effect can be compensated by changing the cold dark matter density, in such a way that $z_{\text {rec }}$ remains fixed, see Ref. [26]. Therefore, due to the degeneracy with the cold dark matter component, the change induced at low $\ell$ is negligible and the largest impact of $N_{\text {eff }}$ on the CMB temperature anisotropies comes from its effect on high multipoles $\ell$, since a higher value of $N_{\text {eff }}$ will induce a drop in power at small scales due to an increased Silk damping. Silk damping refers to the suppression in power of the CMB temperature anisotropies on scales smaller than the photon diffusion length. As a random walk process, the diffusion distance $r_{d}$ will increase as the square root of time and therefore a higher expansion rate $H(z)$ (caused by a higher $N_{\text {eff }}$ ) will decrease $r_{d}$, decreasing therefore the damping. However, in order to keep the acoustic scale $\theta_{A}$ fixed, the comoving

TABLE III. Constraints on the sum neutrino masses $\sum m_{\nu}$ from WMAP data and different possible combinations of galaxy clustering measurements from SDSS-II [interpreted either in the form of matter power spectrum measurements (MPK) or in the form of BAO signals (BAO)], HST and SNLS3 data sets. We have fixed the number of massive neutrinos to 3.046.

\begin{tabular}{lcccccccc}
\hline \hline & & WMAP+ & WMAP+ & WMAP + MPK+ & WMAP + BAO+ & WMAP + MPK+ & WMAP + BAO+ \\
& & MPK & BAO & HST & HST & SNLS3 & SNLS3 \\
\hline$\sum m_{\nu}(\mathrm{eV})$ & $68 \%$ C.L. & $<0.37$ & $<0.29$ & $<0.16$ & $<0.22$ & $<0.22$ & $<0.27$ \\
& 95\% C.L. & $<0.63$ & $<0.52$ & $<0.32$ & $<0.42$ & $<0.42$ & $<0.47$ \\
\hline \hline
\end{tabular}


TABLE IV. Constraints on $\sum m_{\nu}$ from WMAP and different possible combinations of the new $\mathrm{BAO}$ data explored here $\left(\mathrm{BAO}_{2012}\right)$, HST and SNLS3 data sets. We have fixed the number of massive neutrinos to 3.046.

\begin{tabular}{lcccc}
\hline \hline & & $\mathrm{WMAP}+\mathrm{BAO}_{2012}$ & $\mathrm{WMAP}+\mathrm{BAO}_{2012}+\mathrm{HST}$ & $\mathrm{WMAP}^{2} \mathrm{BAO} 2012+\mathrm{SNLS3}$ \\
\hline$\sum m_{\nu}(\mathrm{eV})$ & $68 \%$ C.L. & $<0.26$ & $<0.19$ & $<0.25$ \\
& $95 \%$ C.L. & $<0.48$ & $<0.38$ & $<0.47$ \\
\hline \hline
\end{tabular}

angular $r_{\theta}\left(z_{\text {rec }}\right)$ distance at decoupling should scale in the same way as $r_{s}\left(z_{\text {rec }}\right)$ does [i.e., as $\left.1 / H(z)\right]$ and the angular scale of the Silk damping $\theta_{d}=r_{d} / r_{\theta}\left(z_{\text {rec }}\right)$ will grow as $\sqrt{H}$. Consequently, the damping will be increased when $N_{\text {eff }}$ does due to the higher expansion rate $H(z)$, see Ref. [26].

This first nonstandard scenario is described by the following set of parameters:

$$
\left\{\omega_{b}, \omega_{c}, \Theta_{s}, \tau, n_{s}, \log \left[10^{10} A_{s}\right], N_{\text {eff }}\right\}
$$

However, we know from neutrino oscillation experiments that neutrinos have nonzero masses and mixings. In fact, a recent neutrino oscillation analysis has shown that models with three active plus two sterile light (sub-eV) neutrino species provide a very good fit to short baseline data [39]. Therefore the previous minimal nonstandard scenario with $N_{\text {eff }}$ massless neutrinos should be enlarged to accommodate active plus sterile neutrino masses and mixings. The last scenario explored here therefore consists

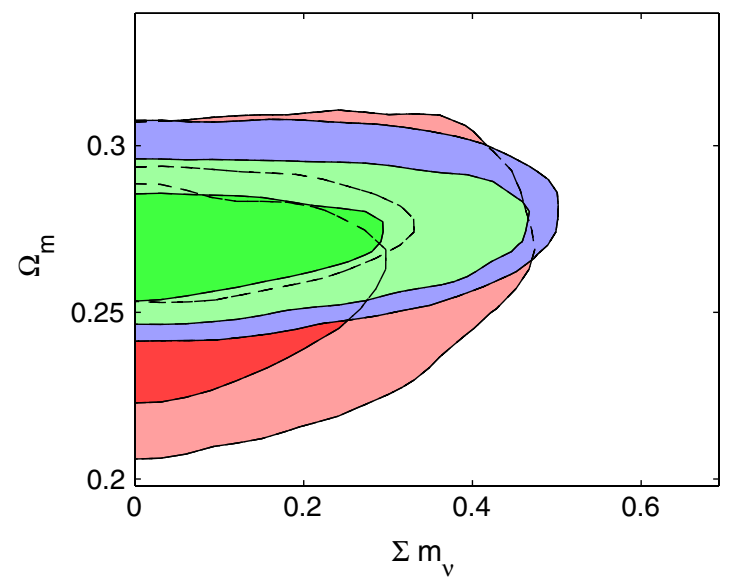

FIG. 2 (color online). The largest red contours show the $68 \%$ and $95 \%$ C.L. constraints in the $\left(\sum m_{\nu}, \Omega_{m}\right)$ plane from WMAP plus HST data sets. The blue contours show the results from the combination of WMAP, HST, and galaxy clustering measurements from SDSS-II interpreted in the form of BAO signals, while the smallest green contours depict the results from the combination of WMAP, HST, and $\mathrm{BAO}_{2012}$ data sets. The errors on the $\Omega_{m}$ parameter are significantly improved when considering either the SDSS-II BAO or the $\mathrm{BAO}_{2012}$ measurements. Also, due to the higher mean value of $\Omega_{m}$ when adding BAO information to the WMAP and HST measurements, a slightly higher neutrino mass is allowed. of $N_{\text {eff }}$ massive neutrino species, each with equal ${ }^{1}$ mass $m_{\nu}$. The effects of these two parameters on the observables have been summarized above. While the highest sensitivity to the neutrino masses comes from large scale structure information, and the highest $N_{\text {eff }}$ sensitivity comes from CMB measurements, there will be significant covariance between the two parameters and we explore this degeneracy in Sec. IV B 2. In this last nonstandard scenario, the set of parameters involved in the study is

$$
\left\{\omega_{b}, \omega_{c}, \Theta_{s}, \tau, n_{s}, \log \left[10^{10} A_{s}\right], N_{\mathrm{eff}}, \sum m_{\nu}\right\} .
$$

For our numerical analyses, we have used the Boltzmann CAMB code [40] and extracted cosmological parameters from current data using a Monte Carlo Markov chain (MCMC) analysis based on the publicly available MCMC package Cosmomc [41]. Table I specifies the priors considered on the different cosmological parameters. ${ }^{2}$ Our neutrino mass prior is cast in the form of a (uniform) prior on the neutrino density fraction $f_{\nu}=$ $\Omega_{\nu} / \Omega_{\mathrm{DM}}$, where $\Omega_{\nu}$ is the ratio of the neutrino energy density over the critical density at redshift zero, and $\Omega_{\mathrm{DM}}$ is the same ratio, but for the total dark matter density, which includes cold dark matter and neutrinos.

We restrict ourselves to a $\Lambda$ CDM cosmology in the different scenarios described above.

\section{DATA}

Our baseline data set is the seven-year WMAP data $[2,42]$ (temperature and polarization) with the routine for computing the likelihood supplied by the WMAP team. We then also add CMB data from the South Pole Telescope (SPT) [23], which strongly improve the measurement of the temperature anisotropies on scales $\lesssim 10$ arcmin. In order to address foreground contributions, the Sunyaez Zeldovich (SZ) amplitude $A_{\mathrm{SZ}}$, the amplitude of the clustered point source contribution, $A_{C}$, and the amplitude of the Poisson distributed point source contribution, $A_{P}$, are added as nuisance parameters in the CMB data analyses.

\footnotetext{
${ }^{1}$ Also in the presence of additional species, current data are not sensitive to how the masses are distributed over the species, but only to the sum of the masses and the number of species. This justifies the use of a degenerate mass spectrum.

${ }^{2}$ In the next section we will describe the additional nuisance parameters that we have also added in the analyses. These parameters are related to some cosmological data sets exploited here.
} 
TABLE V. Constraints on $N_{\text {eff }}$ for the massless neutrino scenario from WMAP data, WMAP data and HST measurements of the Hubble constant, WMAP and SPT data and WMAP plus SPT plus SNLS3 data.

\begin{tabular}{cccccc}
\hline \hline & WMAP & WMAP + HST & WMAP + SPT & WMAP + SPT + HST & WMAP + SPT + SNLS3 \\
\hline$N_{\text {eff }}$ & $5.99_{-1.23-3.47}^{+4.01+4.11}$ & $4.19_{-0.36-1.26}^{+0.33+1.30}$ & $4.13_{-0.41-1.30}^{+0.35+1.46}$ & $3.83_{-0.25-0.76}^{+0.21+0.86}$ & $3.51_{-0.35-1.16}^{+0.31+1.25}$ \\
\hline \hline
\end{tabular}

We have followed Ref. [23], applying a Gaussian prior on the amplitude of each of these three foreground terms.

To the WMAP basic data set we add the latest constraint on the Hubble constant $H_{0}$ from the Hubble Space Telescope (HST) [43], and supernova data from the threeyear Supernova Legacy Survey (SNLS3), see Ref. [44]. In the case of SNLS3 data, we add in the MCMC analysis two extra nuisance parameters related to the light curve fitting procedure used to analyze the supernova ( $\mathrm{SN}$ ) data. These parameters characterize the dependence of the intrinsic supernova magnitude on stretch (which measures the shape of the SN light curve) and color [44].

We also consider data from galaxy clustering that we summarize next. We first employ data from SDSS-II (Sloan Digital Sky Survey; [45]) data release 7 (DR7; [46]), analyzing separately the full shape of the halo power spectrum derived from the clustering of luminous red galaxies [47], and the baryon acoustic oscillation (BAO) signal as extracted from the same data set [48]. Then we consider the impact of the most recent measurement of the BAO scale [37] from the CMASS sample in data release 9 (DR9; [33]) of the baryon oscillation spectroscopic survey (BOSS) [34,35], with a median redshift of $z=0.57$. Together with the CMASS DR9 data, we also include the recent measurement of the BAO scale based on a reanalysis (using reconstruction [49]) of the luminous red galaxies (LRG) sample from data release 7 with a median redshift of $z=0.35$ [50], and the measurement of the $\mathrm{BAO}$ signal at a lower redshift $z=0.106$ from the $6 \mathrm{dF}$ galaxy survey $6 \mathrm{dFGS}$ [51]. We will refer to the combination of these three new BAO signals as $\mathrm{BAO}_{2012}$.

\section{RESULTS}

Here we present the constraints from current data on the neutrino thermal abundance $N_{\text {eff }}$ and on the sum of their masses in different scenarios.

\section{A. The standard lore: Three massive neutrinos}

In this section we consider the standard picture, i.e., three massive neutrinos and put constraints on the sum of their masses $\sum m_{\nu}$.

Table II shows the $68 \%$ and $95 \%$ C.L. bounds on $\sum m_{\nu}$ from several combinations of WMAP data with SPT, HST, and SNLS3. These constraints agree quite well with previous analyses in the literature [1]. Note that WMAP alone sets a $95 \%$ C.L. bound of $\sim 1 \mathrm{eV}$ on the sum of neutrino masses. When HST data is added in the analysis the results improve in a very significant way, since HST data helps enormously in breaking the strong degeneracy between $\sum m_{\nu}$ and $H_{0}$, see Fig. 1 . Indeed, without a $H_{0}$ measurement, the change induced in the CMB temperature anisotropies caused by an increase in $\sum m_{\nu}$ can be compensated by a decrease in $H_{0}$. The reason is the following: if $\sum m_{\nu}$ increases, the main effect on the CMB comes from the resulting shift in the distance to last scattering. ${ }^{3}$ While the acoustic peak structure of the CMB data does not leave much freedom in $\omega_{c}$ and $\omega_{b}$, the change in distance can be compensated by lowering $h$ (i.e., $H_{0}$ ). The HST prior on the Hubble parameter will break this strong degeneracy, setting a $\sim 0.4 \mathrm{eV} 95 \%$ C.L. bound on the sum of the three active neutrino masses. A similar result but to a lesser extent arises when combining WMAP and SNLS3 data sets. Note that we follow here a conservative approach avoiding the combination of HST and SNLS3 data, since these two data sets are not totally independent. The addition of SPT data does not help in constraining $\sum m_{\nu}$ : taking into account the marginalization over the additional nuisance parameters, the high $\ell$ multipole data are not sensitive to neutrino mass, nor do they help break degeneracies to improve the neutrino bound and therefore we will not consider the SPT data set in the rest of the analyses presented in this section. Of course this will not be the case when the effective number of neutrino species is added as a free parameter in the analyses, since, in that case, SPT high $\ell$ measurements will become crucial, as the combination of WMAP and HST will not be enough to break the strong degeneracy between $N_{\text {eff }}$ and $\sum m_{\nu}$.

Table III shows the constraints in the standard massive neutrino scenario when SDSS-II galaxy clustering data are added in the analysis. Note that the bounds from geometrical information from the BAO signal are very similar to those obtained from the shape of the galaxy power spectrum measurements, although BAO information provides slightly better constraints on the sum of the three active neutrino masses. These findings agree with previous analyses in the literature, see e.g., Ref. [4]. However, we are considering here a minimal cosmological model, with spatial flatness and a cosmological constant. $\mathrm{BAO} /$ geometrical data will not be as powerful as shape measurements when other cosmological parameters are added in the analysis, since the information contained in the full shape of the matter power spectrum is extremely useful for breaking degeneracies. We will illustrate this effect in

\footnotetext{
${ }^{3} r_{\theta}\left(z_{\mathrm{rec}}\right) \propto \int_{0}^{z_{\mathrm{rec}}} d z\left[\omega_{r} a^{-4}+\omega_{m} a^{-3}+\left(1-\omega_{m} / h^{2}\right)\right]^{-1 / 2}$, with $\omega_{m}=\omega_{b}+\omega_{c}+\omega_{\nu}$.
} 
TABLE VI. Constraints on $N_{\text {eff }}$ from WMAP and different possible combinations of the new BAO data explored here (BAO 2012$)$, HST and SNLS3 data sets.

\begin{tabular}{ccccc}
\hline \hline & $\mathrm{WMAP}+\mathrm{BAO}_{2012}$ & $\mathrm{WMAP}+\mathrm{SPT}+\mathrm{BAO}_{2012}$ & $\mathrm{WMAP}^{2} \mathrm{SPT}+\mathrm{BAO}_{2012}+\mathrm{HST}$ & $\mathrm{WMAP}^{2} \mathrm{SPT}+\mathrm{BAO}_{2012}+\mathrm{SNLS3}$ \\
\hline$N_{\text {eff }}$ & $5.86_{-1.15-3.36}^{+1.07+3.51}$ & $3.48_{-0.36-1.17}^{+0.31+1.27}$ & $3.66_{-0.21-0.69}^{+0.20+0.73}$ & $3.52_{-0.38-1.16}^{+0.31+1.30}$ \\
\hline \hline
\end{tabular}

Sec. IV B 2, where the addition of the number of massive neutrino species $N_{\text {eff }}$ will open up new degeneracies, and the matter power spectrum shape information will be more powerful in resolving them than the geometrical BAO measurements. Note from Table III that if other data sets are included, such as, for instance, a prior on $H_{0}$ from the HST experiment, some degeneracies are broken, and the constraints on $\sum m_{\nu}$ from shape and geometrical SDSS-II measurements of the galaxy distribution become very similar again. In fact, once the HST prior is included, further inclusion of the large scale structure data considered here does not significantly improve the neutrino mass bound in the first place.

Table IV shows the constraints on $\sum m_{\nu}$ with the addition of the new BAO data from the CMASS DR9 BOSS experiment combined with a reanalysis of the LRGDR7 sample and the BAO signal from 6dFGS. Note that the bounds in this minimal cosmological model from the $\mathrm{BAO}_{2012}$ data set combination are slightly better, albeit very close to those obtained with BAO SDSS-II measurements. Note from Tables III and IV that the combination of WMAP and HST data with old BAO measurements gives a bound on $\sum m_{\nu}$ which is worse than the one arising from the combination of WMAP plus HST alone. The errors on the $\Omega_{m}$ parameter are significantly improved when considering either the SDSS-II BAO or the $\mathrm{BAO}_{2012}$ measurements, see Fig. 2. The mean value of $\Omega_{m}$ is also higher when adding BAO information to the WMAP and HST measurements and, consequently, a slightly higher neutrino mass is allowed.

\section{B. Beyond the standard lore: Varying $\boldsymbol{N}_{\text {eff }}$}

\section{Neglecting neutrino mass}

We assume here a scenario with $N_{\text {eff }}$ massless neutrino species. Table $\mathrm{V}$ shows the central values and the $68 \%$ and $95 \%$ C.L. errors on the quantity $N_{\text {eff }}$ for our basic WMAP data set alone and also with other data sets explored here. We observe that WMAP data alone is unable to constrain the value of $N_{\text {eff }}$ but the situation drastically improves when adding either SPT or HST data. The reason for that is because $N_{\text {eff }}$ is highly degenerate with both $H_{0}$ and the dark matter energy density $\Omega_{\mathrm{DM}} h^{2}$ at the multipole range relevant to WMAP CMB measurements: the change induced in the CMB spectrum by $N_{\text {eff }}$ can be compensated by a change in the value of the Hubble constant $H_{0}$. HST data is crucial for breaking the strong degeneracies between $N_{\text {eff }}$ and both the physical amount of matter $\Omega_{\mathrm{DM}} h^{2}$ and the Hubble constant $H_{0}$, therefore, the prior on $H_{0}$ from HST improves significantly the constraints on $N_{\text {eff }}$. In the following section we will see when adding $\sum m_{\nu}$ as a free parameter the errors on $N_{\text {eff }}$ from WMAP plus HST will be much higher, due to the strong degeneracy between the effective number of neutrino species and the sum of the neutrino masses.

SPT data is also very powerful in constraining $N_{\text {eff }}$ due to the information contained at high multipoles $\ell$. As summarized in Sec. II, an increasing value of $N_{\text {eff }}$ has little (big) impact on low (high) multipoles $\ell$ due to an increased Silk damping caused by a higher expansion rate. Therefore, SPT high multipole data helps enormously in constraining the value of $N_{\text {eff }}$. The combination of WMAP, SPT, and HST gives $3.83_{-0.25-0.76}^{+0.21+0.86}$ which deviates $\sim 2 \sigma$ from the expected standard model value for $N_{\text {eff }}$. The combination of CMB measurements with SNLS3 luminosity distance data results in a mean value of $N_{\text {eff }}$ which is only $\sim 1 \sigma$ away from its the standard value.

The addition of the information contained in the matter power spectrum or in the BAO signal from SDSS-II galaxy clustering measurements to our basic WMAP data set does not change significantly the central values nor the errors depicted in Table $\mathrm{V}$ and for the sake of clarity we do not show all the possible combinations here. We only illustrate the case with the addition of the new BAO data from the BOSS DR9 CMASS sample combined with a reanalysis of the LRGDR7 sample and the BAO signal from 6dFGS, see Table VI. The evidence for extra radiation $N_{\text {eff }}>3$ claimed in the literature still remains at the $2 \sigma$ level, in perfect agreement with a recent analysis presented in Ref. [12].

TABLE VII. Constraints on the number of massive neutrino species and on the sum of their masses $\sum m_{\nu}$ from WMAP data alone, WMAP data and HST measurements of the Hubble constant, WMAP and SPT data, WMAP and SNLS3 data, and WMAP plus SPT plus SNLS3 data.

\begin{tabular}{lccccccc}
\hline \hline & & & & WMAP + SPT+ & \multicolumn{2}{c}{ WMAP + SPT+ } \\
& & WMAP & WMAP + HST & WMAP + SPT & HST & WMAP + SNLS3 & SNLS3 \\
\hline$N_{\text {eff }}$ & & $6.18_{-1.18-3.34}^{+3.82+3.82}$ & $5.91_{-0.94-2.44}^{+4.09+4.09}$ & $3.91_{-0.39-1.15}^{+0.30+1.32}$ & $4.19_{-0.30-0.92}^{+0.25+1.02}$ & $5.97_{-1.17-3.28}^{+4.03+4.03}$ & $3.46_{-0.37-1.17}^{+0.32+1.31}$ \\
$\sum m_{\nu}(\mathrm{eV})$ & $68 \%$ C.L. & $<0.90$ & $<1.07$ & $<0.81$ & $<0.46$ & $<0.28$ & $<0.22$ \\
& 95\% C.L. & $<2.02$ & $<2.25$ & $<1.47$ & $<0.90$ & $<0.66$ & $<0.50$ \\
\hline \hline
\end{tabular}


TABLE VIII. Constraints on the number of massive neutrino species and on the sum of their masses $\sum m_{\nu}$ from WMAP data and different possible combinations of galaxy clustering measurements from SDSS-II [interpreted either in the form of matter power spectrum measurements (MPK) or in the form of BAO signals (BAO)], HST and SNLS3 data sets.

\begin{tabular}{|c|c|c|c|c|c|c|c|}
\hline & WMAP + MPK & $\begin{array}{l}\text { WMAP+ } \\
\text { BAO }\end{array}$ & $\begin{array}{l}\text { WMAP+ } \\
\mathrm{BAO}_{2012}\end{array}$ & $\begin{array}{c}\mathrm{WMAP}+\mathrm{MPK}+ \\
\mathrm{SPT}+\mathrm{SNLS} 3\end{array}$ & $\begin{array}{c}\text { WMAP+ } \\
\text { BAOSPT+ } \\
\text { SNLS3 }\end{array}$ & $\begin{array}{l}\text { WMAP+ } \\
\mathrm{BAO}_{2012}+ \\
\mathrm{SPT}+\mathrm{HST}\end{array}$ & $\begin{array}{c}\mathrm{WMAP}+\mathrm{BAO}_{2012}+ \\
\mathrm{SPT}+\mathrm{SNLS} 3\end{array}$ \\
\hline$N_{\text {eff }}$ & $4.89_{-0.95-2.42}^{+0.68+3.18}$ & $6.18_{-1.15-3.53}^{+3.82+3.82}$ & $5.60_{-1.24-3.55}^{+1.14+3.68}$ & $3.63_{-0.32-1.05}^{+0.29+1.14}$ & $3.44_{-0.37-1.14}^{+0.33+1.24}$ & $3.71_{-0.22-0.72}^{+0.20+0.75}$ & $3.44_{-0.37-1.24}^{+0.33+1.20}$ \\
\hline$\sum m_{\nu}(\mathrm{eV}) 68 \%$ C.L. & $<0.51$ & $<0.93$ & $<0.29$ & $<0.27$ & $<0.21$ & $<0.26$ & $<0.25$ \\
\hline $95 \%$ C.L. & $<1.07$ & $<2.11$ & $<0.59$ & $<0.50$ & $<0.47$ & $<0.51$ & $<0.47$ \\
\hline
\end{tabular}

\section{Including neutrino mass}

We consider here a scenario with $N_{\text {eff }}$ massive species, each with a mass $m_{\nu}$. Table VII shows the joint constraints on the sum of neutrino masses $\sum m_{\nu}$ and on the number of massive neutrino species $N_{\text {eff }}$ when HST, SPT, and SNIa data are added to our basic WMAP data set. Note that the addition of HST data to the WMAP basic data set does not help as in the massless neutrino scenario, the reason for that being that $m_{\nu}$ is now a free parameter and it is strongly degenerate with $H_{0}$ (among other parameters, such as the matter energy density). Therefore, a prior on $H_{0}$ is not enough to improve the constraints from WMAP alone. The errors on $N_{\text {eff }}$ are an order of magnitude larger than in the massless neutrino scenario. A similar effect occurs when addressing the neutrino mass bound: in the case where only three active massive neutrinos were considered, the WMAP and HST data combination provided a 95\% C.L. bound $\sum m_{\nu}<0.38 \mathrm{eV}$. The former bound becomes $2.25 \mathrm{eV}$ when $N_{\text {eff }}$ is allowed to vary as well.

High multipole SPT data helps in constraining $N_{\text {eff }}$. SNIa data is very useful with respect to neutrino masses. However, both the mean value of $N_{\text {eff }}$ and its error are very large (due to the strong degeneracy between $N_{\text {eff }}$ and $\sum m_{\nu}$ as we shall see below) unless SPT data is added in the analysis. Such an addition (i.e., WMAP + SPT + SNLS3)

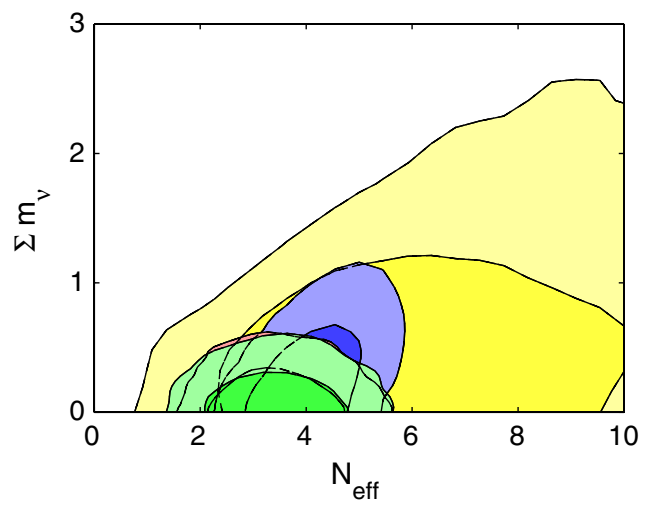

allows one to partially break the strong degeneracy between $\sum m_{\nu}$ and $N_{\text {eff }}$ and it decreases both the mean value of $N_{\text {eff }}$ and its errors significantly. Note from Table VII that the neutrino mass bounds from CMB measurements plus SNIa data are better than those from CMB plus HST measurements. After the addition of SNLS3 measurements, the data combination prefers a lower value of $\Omega_{m}$ which, in turn, leads to a smaller value of $\sum m_{\nu}$.

Table VIII shows the constraints in the massive neutrino scenario when data from galaxy clustering measurements are added in the analysis. When considering SDSS-II measurements, the bounds are stronger (about a factor of 2) if the information from galaxy clustering is exploited as shape constraints (i.e., power spectrum measurements) rather than as geometrical measurements (i.e., BAO signals), the reason for that being the presence of more degeneracies involved, as previously explained. When other data sets are included, some degeneracies are broken, and the constraints from shape and geometrical SDSS-II measurements of the galaxy distribution are very similar. We also depict the bounds on the sum of neutrino masses and on the number of massive species when new BAO information from the BOSS DR9 CMASS sample, combined with a reanalysis of the LRGDR7 sample and with the BAO signal from 6dFGS, are added in the analysis.

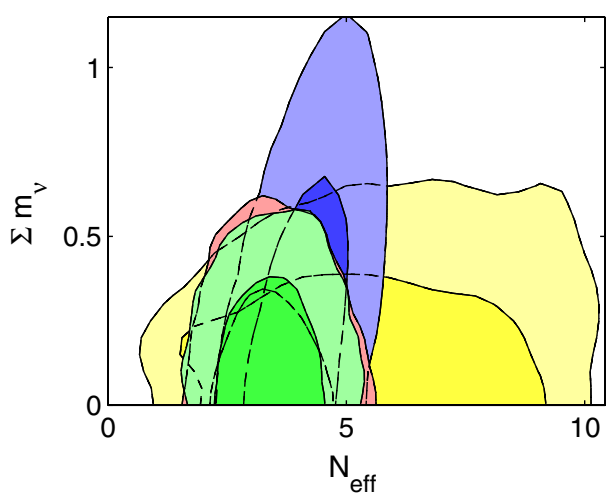

FIG. 3 (color online). Left panel: The largest yellow contours show the $68 \%$ and $95 \%$ C.L. constraints in the $\left(N_{\text {eff }}, \sum m_{\nu}\right)$ plane from our basic WMAP data set combined with old BAO data. The blue contours show the results from the combination of WMAP, SPT, and HST measurements, while the red contours depict the results from the combination of WMAP, SPT, and SNLS3 measurements. Finally, the green contour denotes the constraints from the combination of WMAP, SPT, SNLS3, and BAO data. Right panel: As in the left panel but using recent $\mathrm{BAO}_{2012}$ data. The red and green contours are almost identical. 
Note that these new BAO data sets are more powerful than the previous $\mathrm{BAO}$ data set because they combine $\mathrm{BAO}$ measurements at several redshifts, rely on a larger volume, and include an improvement of the original SDSS-II BAO measurement. Because of this, the $\mathrm{BAO}_{2012}$ constraints are even better than those obtained with the full matter power spectrum of SDSS-II.

The strong degeneracy between $N_{\text {eff }}$ and $\sum m_{\nu}$ is illustrated in Fig. 3, where we show the combination of WMAP with new and old BAO data sets as well as the combination of CMB data with HST or SNLS3 measurements. Finally, we also show the combination of CMB, BAO (both new and old data sets), and SNLS measurements. As in the massless neutrino case, the addition of new $\mathrm{BAO}_{2012}$ data to $\mathrm{CMB}$ and HST measurements makes the value of $N_{\text {eff }}$ (marginally) consistent with the standard expectation within $2 \sigma$, in agreement with previous analyses in the literature. Therefore the evidence for $N_{\text {eff }}>3$ still persists when considering massive neutrino species. The $95 \%$ C.L. constraints on the effective number of massive neutrino species and on the sum of their masses from CMB measurements, SNLS3 and $\mathrm{BAO}_{2012}$ data are $3.44_{-1.24}^{+1.20}$ and $\sum m_{\nu}<0.47 \mathrm{eV}$, respectively. The former bounds translate into $3.71_{-0.72}^{+0.74}$ and $\sum m_{\nu}<0.51 \mathrm{eV}$ when CMB, HST, and $\mathrm{BAO}_{2012}$ measurements are considered. The bounds and the errors found in this work on both $\sum m_{\nu}$ and $N_{\text {eff }}$ are similar to those presented in a recent study presented in Ref. [12], where a preference for $N_{\text {eff }}>3$ at a $2 \sigma$ level has been presented.

\section{CONCLUSIONS}

We have presented constraints on several neutrino-extra radiation scenarios with the most recent and available cosmological data. The data sets explored here include the recent measurement of the BAO feature in the DR9 CMASS sample of the BOSS experiment. Three scenarios have been carefully examined: one standard scenario with three massive neutrino species, and two nonstandard scenarios with $N_{\text {eff }}$ massless/massive neutrino species. In each scenario we have carefully explored the different existing degeneracies and the impact of the different data sets exploited here, both separately and combined. In the standard scenario where only three active neutrinos are considered, the combination of CMB WMAP plus HST data helps enormously in breaking the strong degeneracy between $\sum m_{\nu}$ and the Hubble parameter $H_{0}$ that exists with CMB data only, and the addition of further cosmological data sets does not improve significantly the limit on $\sum m_{\nu}$. In this standard scenario, WMAP data plus geometrical information from BAO signals gives similar results to those obtained from WMAP data plus the measurements of the full shape of the matter power spectrum.

In the nonstandard scenario with $N_{\text {eff }}$ massless neutrinos, the combination of WMAP and HST data is also able to break the strong degeneracies between the effective number of neutrino species and other cosmological parameters, such as the Hubble parameter and the physical dark matter energy density. SPT high multipole CMB data is also extremely useful in constraining the effective number of neutrino species, due to the increase in the Silk damping effect at small scales induced in the case of $N_{\text {eff }}>3$. The addition of SDSS-II galaxy clustering dataor recent BAO measurements does not improve the constraints on $N_{\text {eff }}$ in the massless neutrino scenario. We obtain $N_{\text {eff }}=3.66_{-0.21-0.69}^{+0.20+0.73}$ when combining the former BAO data set with CMB and HST measurements. Therefore, the cosmological evidence for $N_{\text {eff }}>3$ claimed often in the literature gets still remains when considering these new data sets, and the value of $N_{\text {eff }}$ very close to the standard model expectations $\left(N_{\text {eff }}=3.04\right)$, being the discrepancy at the $2 \sigma$ level.

In the most general nonstandard scenario explored here, the strong degeneracy existing among the two neutrino parameters $\sum m_{\nu}$ and $N_{\text {eff }}$ makes mandatory the combination of several data sets. The constraints from the combination of WMAP and HST measurements become 1 order of magnitude weaker than in the previous two scenarios ( $N_{\text {eff }}$ fixed or $m_{\nu}$ fixed), although if more data sets are added in the analysis, the situation improves significantly. Given that we know the sum of neutrino masses is nonzero, this means that constraining the number of neutrino species while neglecting the sum of their masses could lead to completely wrong results, and thus in general caution is advised. In the reverse scenario, i.e., constraining $m_{\nu}$ while keeping $N_{\text {eff }}$ fixed, the same danger exists, but the major difference is that $N_{\text {eff }}$ may well be equal to its standard model value, while we know $\Sigma m_{\nu}$ is nonzero.

To summarize the result with our maximal combination of data sets, the joint constraints on the effective number of massive neutrino species and on the sum of their masses from $\mathrm{CMB}$, supernovae and the new BAO data are $3.71_{-0.72}^{+0.75}$ and $\sum m_{\nu}<0.51 \mathrm{eV}$, respectively (both at the 95\% C.L.). Near future cosmological data, as CMB measurements from the Planck mission, combined with other probes, are expected to settle the issue of whether there exists cosmological compelling evidence for extra radiation.

\section{ACKNOWLEDGMENTS}

We gratefully acknowledge Antonio Cuesta for providing the modified version of COSMOMC with the recent BAO measurements included. We also thank Signe RiemerSørensen and Chris Blake for their help with the cross comparison of results. O.M. is supported by the Consolider Ingenio Project No. CSD2007-00060, by PROMETEO/2009/116, by the Spanish Ministry Science Project No. FPA2011-29678 and by the ITN Invisibles PITN-GA-2011-289442. Part of the research described in this paper was carried out at the Jet Propulsion Laboratory, California Institute of Technology, under a contract with the National Aeronautics and Space Administration. 
[1] R. de Putter, O. Mena, E. Giusarma, S. Ho, A. Cuesta, H.-J. Seo, A. Ross, M. White et al., Astrophys. J. 761, 12 (2012).

[2] E. Komatsu et al. (WMAP Collaboration), Astrophys. J. Suppl. Ser. 192, 18 (2011).

[3] B. A. Reid, L. Verde, R. Jimenez, and O. Mena, J. Cosmol. Astropart. Phys. 01 (2010) 003.

[4] J. Hamann, S. Hannestad, J. Lesgourgues, C. Rampf, and Y. Y. Y. Wong, J. Cosmol. Astropart. Phys. 07 (2010) 022.

[5] M. Sorel, J. M. Conrad, and M. Shaevitz, Phys. Rev. D 70, 073004 (2004).

[6] A. Aguilar et al. (LSND Collaboration), Phys. Rev. D 64, 112007 (2001).

[7] J. Hamann, S. Hannestad, G. G. Raffelt, I. Tamborra, and Y. Y. Y. Wong, Phys. Rev. Lett. 105, 181301 (2010).

[8] E. Giusarma, M. Corsi, M. Archidiacono, R. de Putter, A. Melchiorri, O. Mena, and S. Pandolfi, Phys. Rev. D 83, 115023 (2011).

[9] J. Hamann, S. Hannestad, G. G. Raffelt, and Y. Y. Y. Wong, J. Cosmol. Astropart. Phys. 09 (2011) 034.

[10] E. Giusarma, M. Archidiacono, R. de Putter, A. Melchiorri, and O. Mena, Phys. Rev. D 85, 083522 (2012).

[11] S. Joudaki, K. N. Abazajian, and M. Kaplinghat, arXiv:1208.4354.

[12] S. Riemer-Sorensen, D. Parkinson, T. Davis, and C. Blake, arXiv:1210.2131.

[13] S. Hannestad, A. Mirizzi, G. G. Raffelt, and Y. Y. Y. Wong, J. Cosmol. Astropart. Phys. 08 (2010) 001; A. Melchiorri, O. Mena, and A. Slosar, Phys. Rev. D 76, 041303 (2007).

[14] W. Fischler and J. Meyers, Phys. Rev. D 83, 063520 (2011).

[15] T. L. Smith, E. Pierpaoli, and M. Kamionkowski, Phys. Rev. Lett. 97, 021301 (2006).

[16] P. Binetruy, C. Deffayet, U. Ellwanger, and D. Langlois, Phys. Lett. B 477, 285 (2000); T. Shiromizu, K. i. Maeda, and M. Sasaki, Phys. Rev. D 62, 024012 (2000); V. V. Flambaum and E. V. Shuryak, Europhys. Lett. 74, 813 (2006).

[17] E. Calabrese, D. Huterer, E. V. Linder, A. Melchiorri, and L. Pagano, Phys. Rev. D 83, 123504 (2011).

[18] M. Blennow, E. Fernandez-Martinez, O. Mena, J. Redondo, and P. Serra, J. Cosmol. Astropart. Phys. 07 (2012) 022.

[19] G. Mangano, A. Melchiorri, O. Mena, G. Miele, and A. Slosar, J. Cosmol. Astropart. Phys. 03 (2007) 006.

[20] J. Hamann, S. Hannestad, G. G. Raffelt, and Y. Y. Y. Wong, J. Cosmol. Astropart. Phys. 08 (2007) 021.

[21] J. Hamann, J. Cosmol. Astropart. Phys. 03 (2012) 021.

[22] K. M. Nollett and G. P. Holder, arXiv:1112.2683.

[23] R. Keisler et al., Astrophys. J. 743, 28 (2011).

[24] J. Dunkley et al., Astrophys. J. 739, 52 (2011).

[25] M. Archidiacono, E. Calabrese, and A. Melchiorri, Phys. Rev. D 84, 123008 (2011).
[26] Z. Hou, R. Keisler, L. Knox, M. Millea, and C. Reichardt, arXiv:1104.2333.

[27] T. L. Smith, S. Das, and O. Zahn, Phys. Rev. D 85, 023001 (2012).

[28] M. Archidiacono, E. Giusarma, A. Melchiorri, and O. Mena, Phys. Rev. D 86, 043509 (2012).

[29] Y. I. Izotov and T.X. Thuan, Astrophys. J. 710, L67 (2010).

[30] G. Mangano, G. Miele, S. Pastor, O. Pisanti, and S. Sarikas, J. Cosmol. Astropart. Phys. 03 (2011) 035.

[31] A. Melchiorri, O. Mena, S. Palomares-Ruiz, S. Pascoli, A. Slosar, and M. Sorel, J. Cosmol. Astropart. Phys. 01 (2009) 036.

[32] M. A. Acero and J. Lesgourgues, Phys. Rev. D 79, 045026 (2009).

[33] C. P. Ahn et al. (SDSS-III Collaboration), Astrophys. J. Suppl. Ser. 203, 21 (2012).

[34] D. Schlegel et al. (SDSS-III Collaboration), arXiv:0902.4680.

[35] K. S. Dawson et al., arXiv:1208.0022 [Astron. J. (to be published)].

[36] D. J. Eisenstein et al. (SDSS Collaboration), Astron. J. 142, 72 (2011).

[37] L. Anderson et al., arXiv:1203.6594.

[38] R. Jimenez, T. Kitching, C. Pena-Garay, and L. Verde, J. Cosmol. Astropart. Phys. 05 (2010) 035.

[39] J. Kopp, M. Maltoni, and T. Schwetz, Phys. Rev. Lett. 107, 091801 (2011).

[40] A. Lewis, A. Challinor, and A. Lasenby, Astrophys. J. 538, 473 (2000).

[41] A. Lewis and S. Bridle, Phys. Rev. D 66, 103511 (2002).

[42] D. Larson et al., Astrophys. J. Suppl. Ser. 192, 16 (2011).

[43] A. G. Riess, L. Macri, S. Casertano, H. Lampeitl, H. C. Ferguson, A. V. Filippenko, S. W. Jha, W. Li, and R. Chornock, Astrophys. J. 730, 119 (2011); 732, 129(E) (2011).

[44] A. Conley et al., Astrophys. J. Suppl. Ser. 192, 1 (2011).

[45] D. G. York et al., Astron. J. 120, 1579 (2000).

[46] K. N. Abazajian et al., Astrophys. J. Suppl. Ser. 182, 543 (2009).

[47] S. Roychowdhury, J. N. Chengalur, A. Begum, and I. D. Karachentsev, Mon. Not. R. Astron. Soc. 404, 60 (2010).

[48] W. J. Percival et al. (SDSS Collaboration), Mon. Not. R. Astron. Soc. 401, 2148 (2010).

[49] D. J. Eisenstein, H.J. Seo, E. Sirko, and D. N. Spergel, Astrophys. J. 664, 675 (2007).

[50] N. Padmanabhan, X. Xu, D. J. Eisenstein, R. Scalzo, A. J. Cuesta, K. T. Mehta, and E. Kazin, arXiv:1202.0090.

[51] F. Beutler, C. Blake, M. Colless, D. H. Jones, L. StaveleySmith, L. Campbell, Q. Parker, W. Saunders, and F. Watson, Mon. Not. R. Astron. Soc. 416, 3017 (2011). 